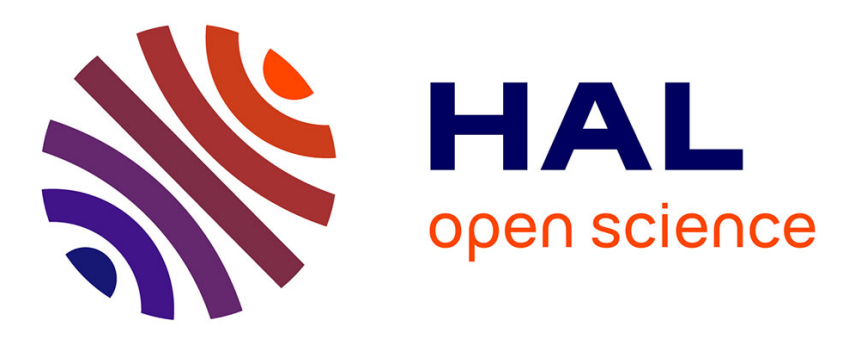

\title{
Hemi-ellipsoidal microlensed fiber based on polishing and polymer technology
}

Nadjiba Boulaiche, Philippe Rochard, Assia Guessoum, Nacer-Eddine

Demagh, Monique Thual

\section{To cite this version:}

Nadjiba Boulaiche, Philippe Rochard, Assia Guessoum, Nacer-Eddine Demagh, Monique Thual. Hemi-ellipsoidal microlensed fiber based on polishing and polymer technology. Engineering Research Express, 2021, 3 (4), pp.045051. 10.1088/2631-8695/ac3a58 . hal-03498608

\section{HAL Id: hal-03498608 \\ https://hal.science/hal-03498608}

Submitted on 21 Dec 2021

HAL is a multi-disciplinary open access archive for the deposit and dissemination of scientific research documents, whether they are published or not. The documents may come from teaching and research institutions in France or abroad, or from public or private research centers.
L'archive ouverte pluridisciplinaire HAL, est destinée au dépôt et à la diffusion de documents scientifiques de niveau recherche, publiés ou non, émanant des établissements d'enseignement et de recherche français ou étrangers, des laboratoires publics ou privés. 
PAPER • OPEN ACCESS

\section{Hemi-ellipsoidal microlensed fiber based on polishing and polymer technology}

To cite this article: Nadjiba Boulaiche et al 2021 Eng. Res. Express 3045051

View the article online for updates and enhancements.
You may also like

Efficient Reformulation of Solid Phase Diffusion in Electrochemical-Mechanical Coupled Models for Lithium-Ion Batteries: Effect of Intercalation Induced Stresses Sumitava De, Bharatkumar Suthar, Derek Rife et al.

A Comprehensive Model of the Meteoroid

Environment around Mercury Diego Janches

- Experimental analysis of surface finishing properties in magnetically assisted abrasive finishing of ASTM B16 brass Palwinder Singh and Lakhvir Singh 


\section{Engineering Research Express}

PAPER

CrossMark

\section{Hemi-ellipsoidal microlensed fiber based on polishing and polymer technology}

RECEIVED

7 September 2021

REVISED

8 November 2021

ACCEPTED FOR PUBLICATION

16 November 2021

PUBLISHED

14 December 2021

Original content from this

work may be used under

the terms of the Creative

Commons Attribution 4.0

licence.

Any further distribution of this work must maintain

attribution to the

author(s) and the title of

the work, journal citation

and DOI.

\author{
Nadjiba Boulaiche ${ }^{1,2}\left(\mathbb{0}\right.$, Philippe Rochard $^{2}$, Assia Guessoum ${ }^{1}$, Nacer-Eddine Demagh ${ }^{1}$ and \\ Monique Thual ${ }^{2}$ (1) \\ 1 Applied optics laboratory, National Institute of Optics and Precision Mechanics, Ferhat ABBAS Setif 1 University, Setif, Algeria \\ 2 Univ Rennes, CNRS, FOTON Institute-UMR 6082, F-22305, Lannion, France \\ E-mail: nadjiba.boulaiche@univ-rennes1.fr
}

Keywords: hemi-ellipsoidal microlens, laser diode, micro-collimator, mode field diameter (MFD), polishing fiber, polydimethylsiloxane (PDMS), single mode fiber (SMF)

\section{Abstract}

This paper represents a development of a new advanced technology to fabricate and characterize micro-collimators with hemi-ellipsoidal microlenses at single-mode fibers outputs. The proposed method utilizes the controlled mechanical micromachining technique based on the variation of the speed of the fiber around its axis in both $\mathrm{X}$ and $\mathrm{Y}$ directions followed by the injection of a quantity of polydimethylsiloxane (PDMS) to form the hemi-ellipsoidal microlenses. The experimental results show that this technique allows to obtain a wide variety of ellipticity diameters ratios from 0.68 to 0.84 . An elliptical ratio of radii of curvature $\mathrm{Ry} / \mathrm{Rx}$ in a range of 0.51 at 0.86 is also obtained. In this investigation a mode field diameters MFD in an interval between $3.26 \mu \mathrm{m}$ and $9.93 \mu \mathrm{m}$ have been realized. The measurement results demonstrate that the proposed technology allows to fabricate hemi-ellipsoidal microlenses having an MFD ellipticity ratios of about 0.60 to 0.97 in near field promising for micro-collimator suitable to match an elliptical laser beam to the circular one of a fiber.

\section{Introduction}

Evolution and development in the field of fiber optic connectivity, in particular optical coupling end elements, is an essential requirement for improving power coupling efficiency between optoelectronic devices, especially laser diodes and optical fibers. The use of Single-Mode Fiber (SMF), in general, has led to the need for the integration of interconnection devices that meet requirements for optimal optical transfer rates of signal in fiber-optic communication systems [1]. The optical beam emitted by a laser diode (LD), being of asymmetrical section, whereas a SMF has a circular geometric section, leads to a low coupling efficiency for high ellipticity laser beams [2]. When coupling a LD to a fiber, the coupling losses are mainly due to the difference in geometrical cross-sections and mismatching of the mode field diameters (MFD); a correction is therefore required.

The integration of asymmetric microlenses at the fiber output is a common approach to achieve better mode matching between anamorphic fibers [3]. The main advantage of elliptical microlenses over spherical microlenses, despite it may require a more advanced fabrication process, is the conversion of the elliptical waist to a circular waist for a Gaussian beam [4]. Several interesting asymmetric microlens structures have been integrated, such as an up-tapered wedge-shaped fiber endface [5], an asymmetric hyperbolic fiber microlens [6,7], an anamorphic microlens [8], a wedge-shaped fiber endface (WSFEF) [9-11], a quadrangular pyramid fiber shaped endface (QPSFE) [12], conical wedge ends (CWSFE) [13, 14], and asymmetric elliptic-cone-shaped microlens (AECSM) [3].

Double variable curvature microlenses [15] and quadric interface microlenses (QIML) [16] are also found. Despite recent research carried out for the optimization of optical coupling using micro-collimators (fiber + microlenses), the problem of coupling losses is still present. The reason is that it is difficult to form microlenses with any different ellipticity ratio of radii of curvatures matching the laser diodes fields ellipticity. However, compared to the previous research techniques on manufacturing of asymmetric fiber microlenses by 


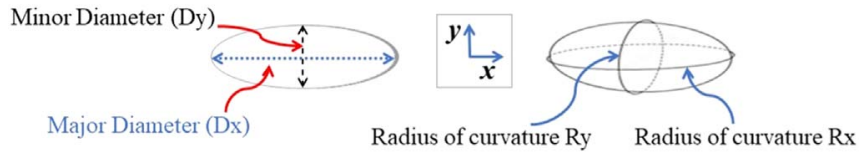

Figure 1. Parameters definitions of the hemi-ellipsoidal microlens, cross section diameters $D x$ and $D y$ and radii of curvature $R x$ and Ry of the microlens.

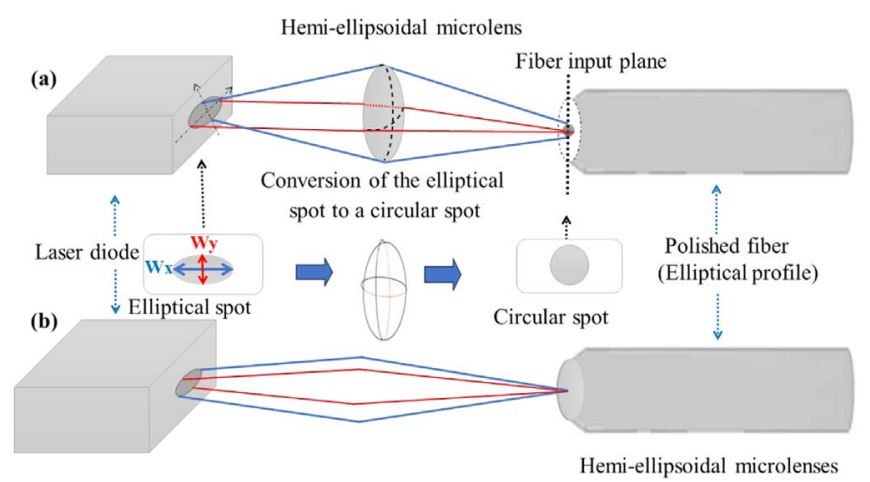

Figure 2. Diagram principle of the coupling between a laser diode and a SMF through the microlens, (a): before assembling microlensfiber, and (b): after assembling. Moreover, the closer the transformed mode field diameters of the LD to the SMF ones, the higher the coupling efficiency.

multistep processes with complicated fabrication, the novelty of the type of micro-collimator developed in this work consists in the possibility of manufacturing hemi-ellipsoidal microlenses of controlled opto-geometrical parameters with a simple, automated, reproducible process promising to be low expensive to fit the laser diode ones. Thanks to this method it is possible to vary the polishing time and consequently the speed in a symmetrical way for the two axes (x, y).

So the same polishing time gives the same speeds $\mathrm{Vx}$, Vy which will subsequently give us the same ellipticity ratio. Therefore, this novel method allows forming microlenses with different targeted radii of curvatures at a SMF fiber output to fit the ellipticity shape of the laser beam modes to be coupled to the SMF. The advantage of this manufacturing technique is the possibility of controlling the elliptical ratio of the microlenses to adapt the mode of the laser to that of fiber.

In this manuscript, the principle and fabrication process of the hemi-ellipsoidal microlens are first presented. Thus, the offset between the top of the microlens and the fiber core center is measured.

Second, in order to review the functionality of the optical components obtained, the characterization results in near field are presented. The mode field diameter characterization of the microlenses by near field technique is developed at a wavelength of $1.55 \mu \mathrm{m}$. The far-field measurement method is also described. Geometric measurements with an optic microscope (radius of curvature, diameter and thickness) are also performed. Finally, before to conclude the asymmetric profiles results are discussed and the mode field diameter (MFD) characterization in near field is compared to calculations.

\section{Principle of the hemi-ellipsoidal microlens}

The coupling efficiency is the result of the overlap integral of the complexe laser diode transverse field distribution after propagating through the microlens and the transverse field distribution of the SMF fundamental mode at the fiber input plane [17]. It will be increased by matching the mode field diameters of the microlens to a laser or to other components to be coupled.

So, the closer this distribution, the better the coupling efficiency. Most of the time, laser diodes have an output beam, with elliptical cross-section whereas the SMF's beam is circular. The ellipticity ratio $(W y / W x)$ is defined by the ratio between the laser diode waists along the $y$ and $x$ axis. This last condition determines the ellipticity ratio that we aim for the microlens to improve coupling efficiency between the LD to a SMF.

The principle of the original fabrication process is to form hemi-ellipsoidal microlenses of different radii of curvatures $(R y / R x)$ in the transverse $\mathrm{x}$ and $y$ axis of a SMF output as illustrated in figure 1 . This is achieved by controlling the ellipticity ratio of the SMF cross section diameters $(D y / D x)$ before adding a polymer droplet at the fiber output. Then, the ratio between the minor axis $(D y)$ and the major axis $(D x)$ as well as the ratio between 


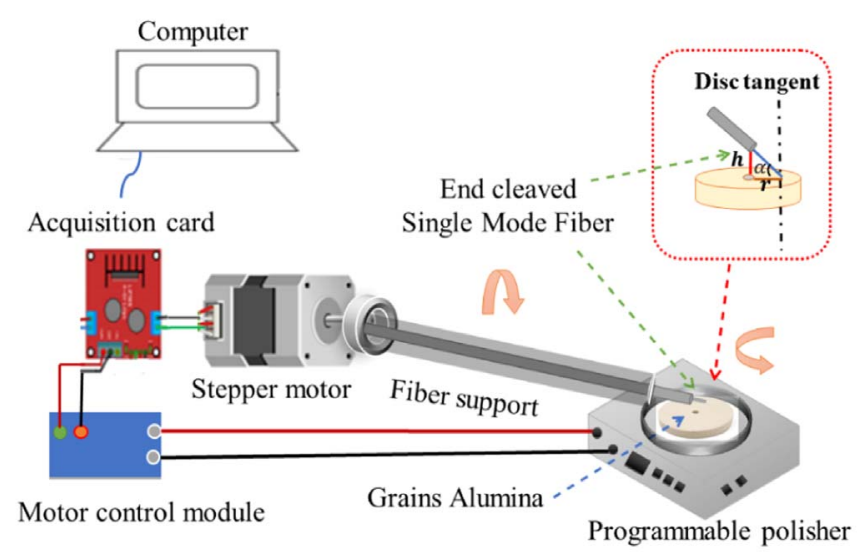

Figure 3. Polishing process for producing end fiber with an elliptical profile.

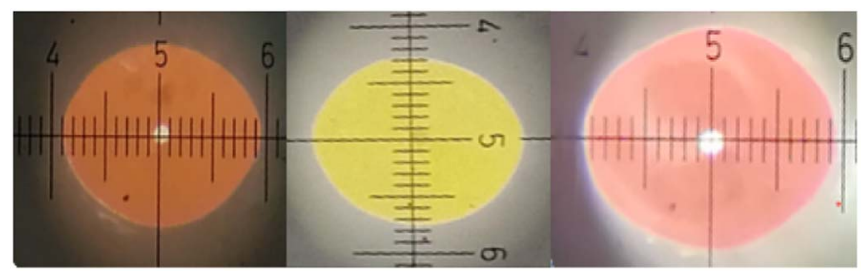

(a)

(b)

(c)

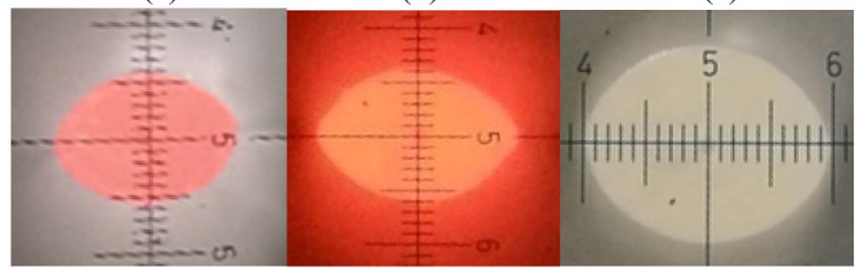

(d)

(e)

(f)

Figure 4. Transverse view of fiber ends with an elliptical profile obtained with the polishing process: figure 4(a) M2, figure 4(b) M9, figure 4(c) M5, figure 4(d) M1, figure 4(e) M4, and figure 4(f) M6. (Scale: 20 graduations correspond to $125 \mu \mathrm{m}$ ).

$(R y)$ and $(R x)$ radii of curvature of the hemi-ellipsoidal microlens are designed to fit the ellipticity ratio $(W y / W x)$ of the laser diode as can be seen in the coupling scheme illustrated in figure 2 . The ellipticity ratios of the diameters of the ellipsoidal microlens is given by (1):

$$
E=D y / D x
$$

\section{Fabrication method}

The manufacturing technique consists in two main steps: first polishing the fiber output in an elliptical transverse section and second depositing polymer to form the hemi-ellipsoidal microlens at the fiber output.

The fibers used in this paper are standard SMFs with a core diameter of $9 \mu \mathrm{m}$ and a cladding diameter of $125 \mu \mathrm{m}$.

The ends of the fiber are prepared before mechanical micromachining by removing the jacket on several $\mathrm{mm}$ $(5 \mathrm{~mm})$, then flat cleaved with a fiber cleaver so that the fiber to be polished is in good condition.

\subsection{Polishing process}

The elliptically shaped fiber is manufactured by first polishing a cleaved fiber to form the asymmetrical shaped cross section end fiber with the desired ellipticity ratio $(D y / D x)$. Polishing is carried out by a programmable automatic polisher using abrasive Alumina $F 1$ grains whose diameter is $1 \mu \mathrm{m}$. A system of rotation of the optical fiber around its axis called revolution, has been adapted to control the rotation speed by an acquisition card DAC multifunction NI, USB-6001. This acquisition card is linked with Stepper motor A4989 type 17PS.M3.6 ${ }^{\circ}$ Hybrid which controls the fiber speed commanded by a program which varies both the rotation times tx, ty of the fiber 
Table 1. Measurements of the position of the fiber core relative to the center of the major axis and the minor axis of the ellipse and measurements of the fiber offseT $( \pm 0.5 \mu \mathrm{m})$.

\begin{tabular}{|c|c|c|c|c|c|c|}
\hline \multirow{2}{*}{$\begin{array}{l}\text { Micro- } \\
\text { lenses }\end{array}$} & \multicolumn{4}{|c|}{ Fiber core position } & \multicolumn{2}{|c|}{ Fiber offset } \\
\hline & Dx1 & Dx2 & Dy1 & Dy2 & Dx & Dy \\
\hline M1 & 51.27 & 51.27 & 36.28 & 36.28 & 0 & 0 \\
\hline M2 & 57.50 & 57.50 & 42.60 & 42.60 & 0 & 0 \\
\hline M3 & 52.86 & 52.86 & 36.28 & 36.28 & 0 & 0 \\
\hline M4 & 58.38 & 58.37 & 40.07 & 40.07 & 0.01 & 0 \\
\hline M5 & 59.95 & 60.74 & 48.12 & 48.12 & 0.79 & 0 \\
\hline M6 & 59.17 & 59.17 & 50.49 & 48.91 & 0 & 1.58 \\
\hline M7 & 49.69 & 48.89 & 35.27 & 35.27 & 0.8 & 0 \\
\hline M8 & 36.86 & 35.26 & 29.65 & 27.25 & 1.60 & 0 \\
\hline M9 & 54.43 & 54.43 & 45.75 & 45.76 & 0 & 0.01 \\
\hline M10 & 43.39 & 43.39 & 35.74 & 35.74 & 0 & 0 \\
\hline
\end{tabular}

$\mathrm{Dx} 1=$ major half diameter $(\mathrm{x}$ direction 1$), \mathrm{Dx} 2=$ major half diameter $(\mathrm{x}$ direction 2), Dy1 = minor half diameter (y direction 1$)$, Dy2 = minor half diameter (y direction 2)

Table 2. Measurement of axis diameters (Dx, Dy), radii of curvature (Rx, Ry), $( \pm 0.50 \mu m)$ and determination of ellipticity ratio (Dy/ $\mathrm{Dx})$ and $(\mathrm{Ry} / \mathrm{Rx})$ of hemi-ellipsoidal microlenses of different thicknesses $\mathrm{T}( \pm 0.50 \mu \mathrm{m})$.

\begin{tabular}{|c|c|c|c|c|c|c|c|}
\hline Micro-lenses & $\begin{array}{c}\mathrm{Dx} \\
(\mu \mathrm{m})\end{array}$ & $\begin{array}{c}\text { Dy } \\
(\mu \mathrm{m})\end{array}$ & Ellipticity ratio Dy/Dx & $\begin{array}{c}\mathrm{Rx} \\
(\mu \mathrm{m})\end{array}$ & $\begin{array}{c}\text { Ry } \\
(\mu \mathrm{m})\end{array}$ & Ellipticity ratio $\mathrm{Ry} / \mathrm{Rx}$ & $\begin{array}{c}\mathrm{T} \\
(\mu \mathrm{m})\end{array}$ \\
\hline M1 & 102.38 & 72.49 & 0.70 & 28.29 & 18.35 & 0.64 & 24.84 \\
\hline M2 & 115 & 85.76 & 0.74 & 55.81 & 40.27 & 0.72 & 14.42 \\
\hline M3 & 105.71 & 72.58 & 0.68 & 39.79 & 23.45 & 0.58 & 25.24 \\
\hline M4 & 116.24 & 80.95 & 0.69 & 46.32 & 30.07 & 0.64 & 16.83 \\
\hline M5 & 120.7 & 96.24 & 0.79 & 51.52 & 37.21 & 0.72 & 11.04 \\
\hline M6 & 118.31 & 99.40 & 0.84 & 43.06 & 28.24 & 0.65 & 32.34 \\
\hline M7 & 98.61 & 71.33 & 0.72 & 40.23 & 22.35 & 0.55 & 13.62 \\
\hline M8 & 72.15 & 56.11 & 0.77 & 28.73 & 14.73 & 0.51 & 30.45 \\
\hline M9 & 108.08 & 91.51 & 0.84 & 57.25 & 49.75 & 0.86 & 15.77 \\
\hline M10 & 86.78 & 71.78 & 0.82 & 41.05 & 33.27 & 0.81 & 29.20 \\
\hline
\end{tabular}

$\mathrm{Dx}=$ diameter ( $\mathrm{x}$ direction), $\mathrm{Dy}=$ diameter ( $\mathrm{y}$ direction $), \mathrm{Rx}=$ radius of curvature ( $\mathrm{x}$ direction $), \mathrm{Ry}=$ radius of curvature $(\mathrm{y}$ direction), $\mathrm{T}=$ Thickness.

around its axis and the polisher rotation by a module with a constant speed (see figure 3). The polishing disc rotation speed is constant all along the process. Fiber inclined angle is given by (2):

$$
\alpha=\tan \frac{h}{r}
$$

where ' $\alpha$ ' is the polishing angle between the fiber axis and the polishing disc, related to $r$ the distance from the rotation center of the polishing disc to the end of the fiber and ' $h$ ' the fiber height relative to the polishing disc rotation center. The rotation speed of the fiber around its axis is varied alternatively from $V y$ to $V x$ every quarter circle leading to an elliptical transverse profile $D y / D x$ of the fiber output depending on the $V y / V x$ speed ratio.

The diagram of mechanical micromachining process for producing the proposed microlensed fibers is illustrated in figure 3 .

The same process is applied on different fibers through varying the rotation speed of the fiber. A series of the images of the asymmetrical end fibers cross section figure 4(a) M2, figure 4(b) M9, figure 4(c) M5, figure 4(d) M1, figure 4(e) M4 and figure 4(f) M6 pointing out different lengths Dx and Dy of major and minor axis forming several ellipticity ratios (table 2), obtained by an optical microscope are pictured in figure 4 . The fibers have the same basic characteristics $9 / 125 \mu \mathrm{m}$ with cladding of different colors; the colors of these views are relative to the plastic cladding when illuminating the fibers to see the cross section after polishing.

The scale of the images shown in this figure with a microscope objective with a magnification of $(10 \mathrm{x} / 0.25)$ is the following: 20 graduations corresponds to $125 \mu \mathrm{m}$ (SMF). 


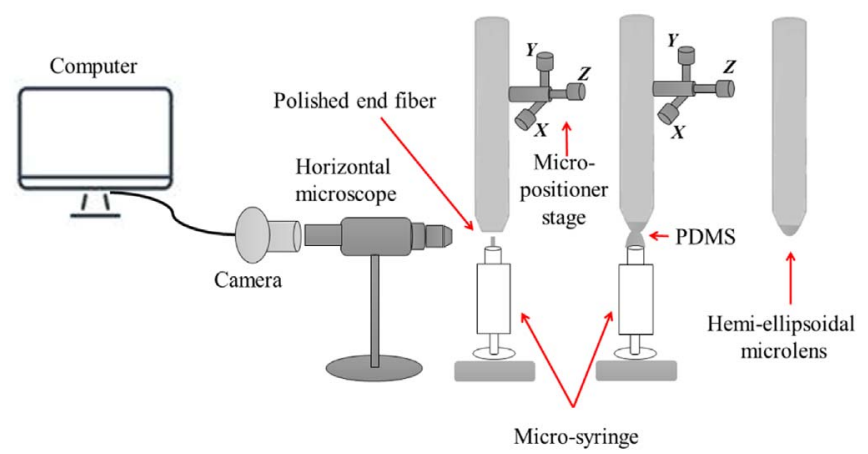

Figure 5. Steps of injection of the PDMS.

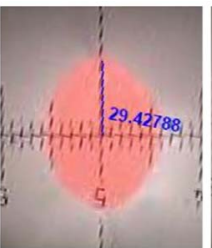

(a)

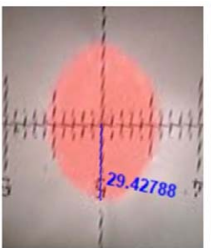

(b)

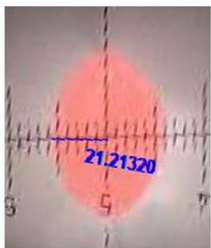

(c)

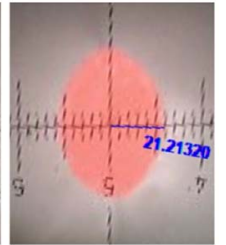

(d)

Figure 6. Measurement (in pixel) of the position of the fiber core relative to the center of the major axis figure and the minor axis of the ellipse for the profile of the M1 microlens.

\subsection{Polymer deposit}

\subsubsection{Choice of material for the deposit}

Waveguide systems made of polymers are known by rapid manufacturing processes, they are commercially available and inexpensive [18]. Polydimethylsiloxane called PDMS is a polymer of the siloxane family. It is a flexible polymer which has a stable conformation as well as a weak evolution of the physical properties. In addition it has a weak intermolecular interaction with the temperature. The refractive index of this polymer is 1.418 at wavelength $\lambda=1.3 \mu \mathrm{m}$.

It is a polymer widely used for the manufacture and prototyping of microfluidic chips. In addition, PDMS has also proven to be an attractive choice for optical components [19-21] thanks to its elastomeric properties, optical transparency, low attenuation and high reliability. Its advantages show its wide use for optical, biological, and biocompatibility applications [19-22].

\subsubsection{PDMS preparation}

The curing agent is necessary to polymerize the PDMS; so these last two were mixed with a quantity of 10: 1 in weight (i.e. $10 \mathrm{~g}$ of PDMS and $1 \mathrm{~g}$ agent). In order to obtain a small radius of curvature, it is necessary to let the PDMS stand for 15 to 30 min so that the PDMS degassed. And it all depends on the ambient temperature (around $25^{\circ}$ ), the mixture is left to stand still in order to increase its viscosity. At this moment, the deposited microdrop will have a small radius of curvature and it takes the same profile as the polished fiber.

\subsubsection{PDMS deposit}

Once the polishing stops, and after the preparation of the mixture (PDMS + curing agent) the fiber is fixed vertically on a micro positioner stage adjustable in $\mathrm{x}, \mathrm{y}$ and $z$ axis. A micro drop of PDMS (polydimethylsiloxane polymer with suitable optical properties) is applied on the polished section, using a flow-controlled microsyringe aligned with the polished fiber and loaded with PDMS by touching the end of fiber with the PDMS (see figure 5). This quantity of PDMS forms the thickness of the microlens which goes from the end of the polished fiber with an elliptical profile to the end of the microlens. Depending on this quantity of PDMS, different thicknesses $T$ can be formed, and for a given quantity of PDMS deposited, the same microlens characteristics are always obtained for a given transverse profile. The process of alignment and deposition of the polymer at the fiber output is monitored and controlled by an acquisition system composed of a horizontal microscope, a CCD camera and a microcomputer [23].

Hemi-ellipsoidal microlenses are thus formed thanks to the surface tension effect (figure 5). After depositing the PDMS on the fiber, the microlens is cured in an oven at a temperature of $100^{\circ} \mathrm{C}$ for one hour. 


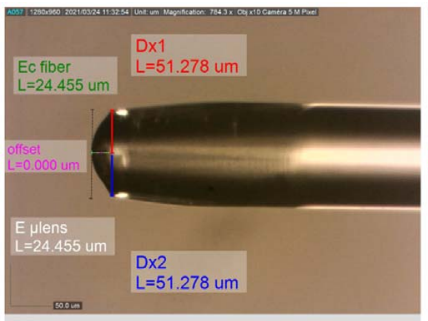

(a)

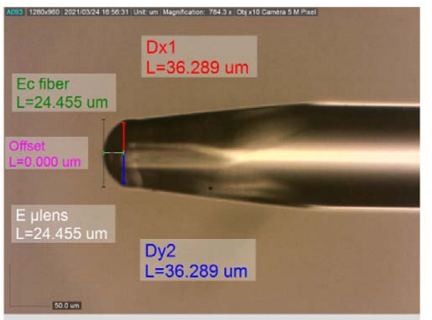

(b)

Figure 7. Measurement of the top of the lens against the fiber core center (M1 microlens).

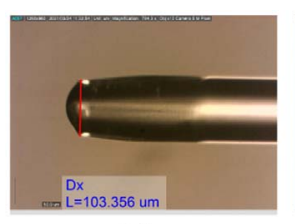

(a)

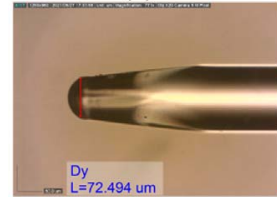

(d)

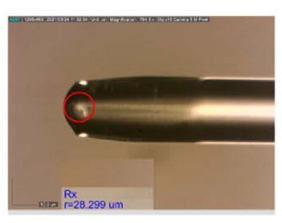

(b)

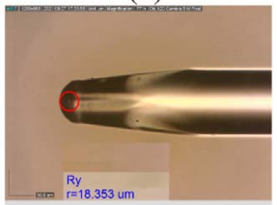

(e)

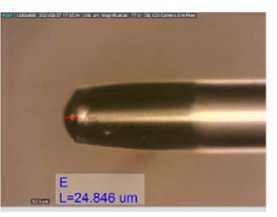

(c)

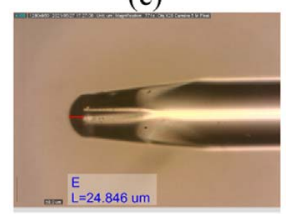

(f)

Figure 8. Cross-sectional view of the hemi-ellipsoidal microlens M1 for the optical fiber after PDMS injection on the elliptical profile of polished fibers (figure 7(c): (a) the major axis measurement (Dx) $\mathrm{L}=103.35 \mu \mathrm{m}$,. (b) radius of curvature measurement (Rx) $\mathrm{r}=28.29 \mu \mathrm{m}$, (d) the minor axis measurement (Dy) $\mathrm{L}=72.49 \mu \mathrm{m},(\mathrm{e})$ radius of curvature measurement $(\mathrm{Ry}) \mathrm{r}=18.35 \mu \mathrm{m},(\mathrm{c})$ and (f) PDMS microlens thickness measurement (T) $\mathrm{L}=24.84 \mu \mathrm{m}$

This completes the realization of an optimized micro-collimator consisting of a combination of optical fibers and microlenses.

\subsection{Offset measurement}

The fabrication of hemi-ellipsoidal microlensed fiber employing polishing technique may produce a fiber offset which is defined by the difference between microlensed center and fiber center. It is important to measure this offset between the top of the microlens and the fiber core center since it may have an influence on the coupling efficiency. For that purpose, the position of the fiber core relative to the center of the major axis and the minor axis of the ellipse is first measured through an optical microscope (Zeiss Axiotech) (column 2,3,4 and 5 of table 1) after the polishing step and before deposition of the polymer (as can be seen in figure 6 below). As an example the measurements for the M1 microlens where the pixel values shown in this figure correspond to the values in $\mu \mathrm{m}$ of the M1 microlens are shown in table 1. The measurements correspond to the major half diameters Dx1:29.42 (pixel) $\rightarrow 51.27 \mu \mathrm{m}, \mathrm{Dx} 2: 29.42$ (pixel) $\rightarrow 51.27 \mu \mathrm{m}$ and minor half diameters Dy1:21.21(pixel) $\rightarrow 36.28 \mu \mathrm{m}$, Dy2: 21.21 (pixel) $\rightarrow 36.28 \mu \mathrm{m}$ of the M1 microlens (fiber profile without microlens). The offset of the M6 microlens is between 0 and 0.78 (table 1). The offset of the position of the fiber core relative to the center of the major axis and the minor axis is comprised in a range of $\pm 0 \mu \mathrm{m}$ to $\pm 0,01 \mu \mathrm{m}$ for microlenses M1,M2,M3,M4, M9, M10,(M5,M7,M8) minor axis (Dy) and M6 major axis (Dx). This is the same range as the core/clad concentricity intrinsic eccentricity specification of standard Single Mode Fiber G652/SMF28 $(<0,5 \mu \mathrm{m})$.

For microlenses (M5, M7, M8) major axis (Dx) and M6 minor axis (Dy) the offset is between $\pm 0.79 \mu \mathrm{m}$ to $\pm 1.6 \mu \mathrm{m}$.

Then an offset could occur during the polymer deposition, but this step is less critical since the droplet is selfcentered by surface tension. The microlens are visualized by illuminating the fiber under the microscope. We measure the distance between the top of the microlens and the top of the fiber by looking at the cross-sectional view of the fiber and measuring the distance between the top of the fiber and the top of the microlens as shown in figure 7 and table 1 . With the polishing technique, whatever the configuration, we all get almost the same error which depends on the quality of the material used. 


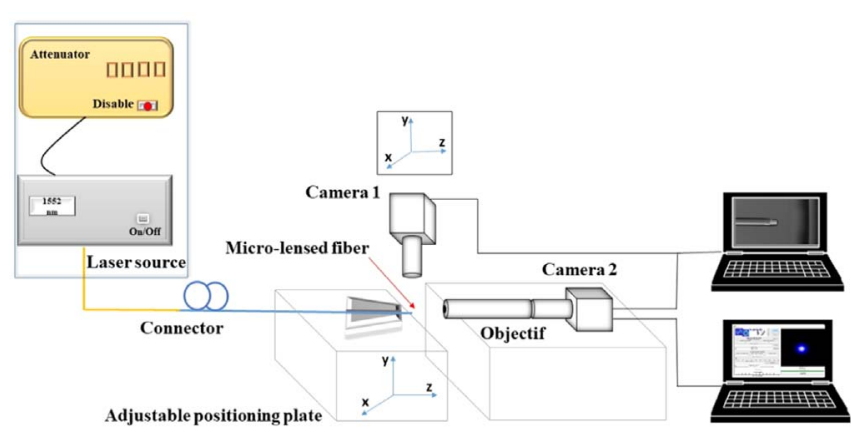

Figure 9. Schematic diagram of the near-field bench.

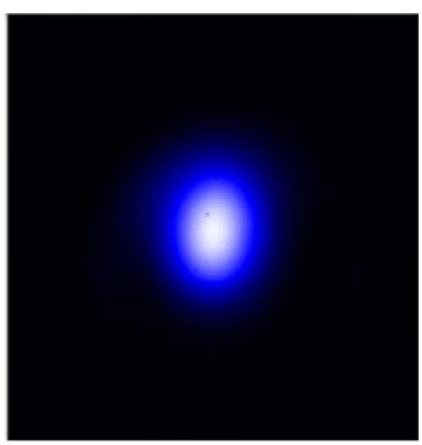

Figure 10. Transverse intensity image at the (M1) microlens output, injected with a $1.55 \mu \mathrm{m}$ emitting laser beam, observed on an IR camera through a microscope objective $(100 \mathrm{x} / \mathrm{NA}=0.9)$ focusing at the waist location.

\section{Geometrical measurements}

Microlenses presented here are designed from micromachined end fibers followed by the injection of the PDMS polymer. Figure 8 shows the side view images while measuring the major and minor axis $D x, D y$, radii of curvature $R x, R y$ and thicknesses $T$ of the hemi-ellipsoidal microlenses formed. This diversity of diameters depends on the variation in the fiber rotation speed around its axis which leads in forming different elliptical ratios of diameters $D y / D x$ ranging from 0.68 at 0.84 (table 2).

Moreover, depending on the amount of PDMS deposited, different thicknesses were obtained which also offers a wide variety of radii of curvature with ratios $R y / R x$ varying between 0.51 and 0.86 (table 2 ).

These values are summarized in table 2, which corresponds to the sectional views in figure 4: each figure corresponds to a microlens referenced in table 2: figure 4(a) M2, figure 4(b) M9, figure 4(c) M5, figure 4(d) M1, figure 4(e) M3, and figure 4(f) M6. Based on the proposed method and by repeating the same polishing process with the same conditions (fiber rotation speed and amount of PDMS deposited), hemi-ellipsoidal microlenses with the same opto-geometric parameters (diameters, radius and thickness) can easily be reproduced.

\section{Mode field characterization results}

The mode field diameter evolution of a Gaussian beam propagating through the microlenses is an important parameter to be characterized. The waist, $2 \mathrm{w}_{0}$, is defined by the smallest mode field diameter of the Gaussian beam where the phase front is plane [17]. The work presented here focuses on the mode field diameters measurements, by the near-field characterization technique; also, the far-field measurement method is presented with examples of measurement of M1 and M5 microlenses at the wavelength of $1.55 \mu \mathrm{m}$.

\subsection{Mode field diameter measurement: Near field}

The near field measurement technique for the MFD consists in: (i) performing the measurement in the Rayleigh area, giving the image of the beam waist at the microlens output via a IR camera (HAMAMATSU InGaAs Model C10633-23) using a large numerical aperture microscope objective (NA $=0,9)$ with a magnification of $(100 x)$ (ii) and visualizing the spot to plot the transverse intensity profile in the image plane [24-26]. This near field 


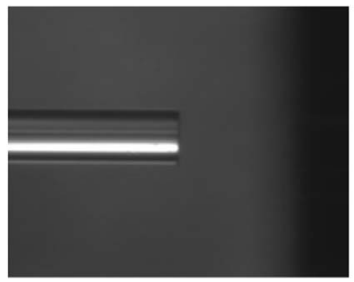

(a)

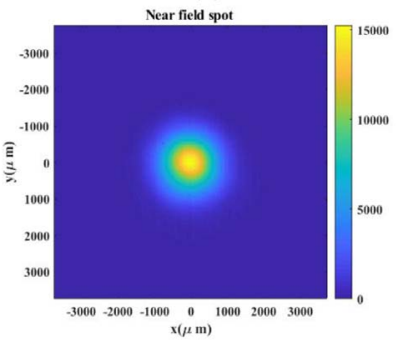

(c)

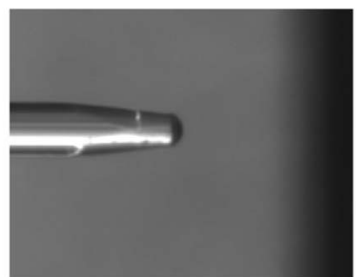

(b)

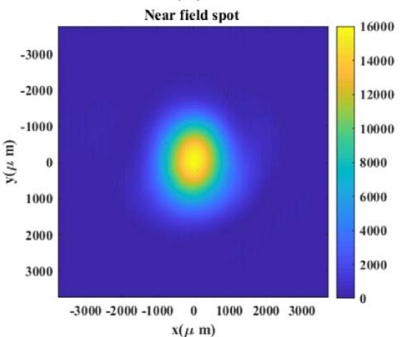

(d)

Figure 11. cross-sectional view of: (a) a cleaved single-mode fiber (SMF), (b) the fiber with PDMS hemi-ellipsoidal M1 microlens surface profile, (c) transverse intensity image of the SMF output, and (d) the M1 micro-collimator output, injected with a $1.55 \mu \mathrm{m}$ emitting laser diode observed on an IR camera though a microscope objective $(100 \mathrm{x} / \mathrm{NA}=0.9)$ focusing at the waist location.

Table 3. Near-field MFD measurement $( \pm 0.50 \mu \mathrm{m})$

\begin{tabular}{lccc}
\hline $\begin{array}{l}\text { Micro- } \\
\text { lenses }\end{array}$ & $\begin{array}{c}\text { MFDx } \\
\text { real }(\mu \mathrm{m})\end{array}$ & $\begin{array}{c}\text { MFDy } \\
\text { real }(\mu \mathrm{m})\end{array}$ & $\begin{array}{c}\text { MFDy/MFDx } \\
\text { ratio }\end{array}$ \\
\hline M1 & 7.23 & 5.95 & 0.82 \\
M2 & 6.52 & 6.38 & 0.97 \\
M3 & 6.24 & 4.82 & 0.77 \\
M4 & 9.93 & 8.08 & 0.81 \\
M5 & 7.09 & 5.67 & 0.79 \\
M6 & 7.80 & 4.68 & 0.60 \\
M7 & 6.81 & 5.10 & 0.74 \\
M8 & 5.10 & 3.26 & 0.63 \\
M9 & 6,58 & 6,24 & 0.94 \\
M10 & 6.95 & 6.66 & 0.95 \\
\hline
\end{tabular}

MFD $=$ Mode field diameter at the waist.

method offers good precision for large mode field diameters (more than $5 \mu$ m diameter) whose divergence angle is quite small and for large numerical aperture (NA) objectives, which is the case in this work.

The manipulation used for this measurement consists of: a $1.55 \mu \mathrm{m}$ laser source launched into a microlensed fiber. The microlensed fiber output beam is observed on the IR camera through the objective $(100 \mathrm{x} / \mathrm{NA}=0.9)$. The principle and the experimental device relating to these measurement techniques are shown in figure 9 . The near-field spot of the hemi-ellipsoidal microlens captured by the IR camera is imaged in the figure (10). This measurement requires prior calibration against the spot of an SMF.A calibration of the hemi-elliptical microlenses mode field diameters is carried out by comparing to a mode field diameter of an SMF (10.5 \pm $0.50 \mu \mathrm{m}$ ) at a wavelength of $1.55 \mu \mathrm{m}$, which normally has a circular profile, see figures 11 (a) and (c).

The transverse intensity profile of the PDMS fabricated hemi-ellipsoid microlens and of the laser beam are shown in figure 11(b) and (d). The beam waist of each microlens is measured in near field.

The real values of MFD at the waist ( $2 \mathrm{w}$ ) of these microlenses as well as their $\mathrm{Wy} / \mathrm{Wx}$ ratios is then found and represented in table 3 .

The spot emerging from the hemi-ellipsoidal microlens has clearly an elliptical shape as can be seen in figures 10 and 11 .

The intensity profile of the elliptical microlenses obtained by this measurement techniques makes it possible to have the intensity at any point by the near field method.

Near field measurement technique is the easiest and fastest technique used to measure spot size.

Figure 12 shows the near field scan and Gaussian fit for SMF (figure12(a)) and for hemi-ellipsoidal microlens (M1) (figure12(b)). 


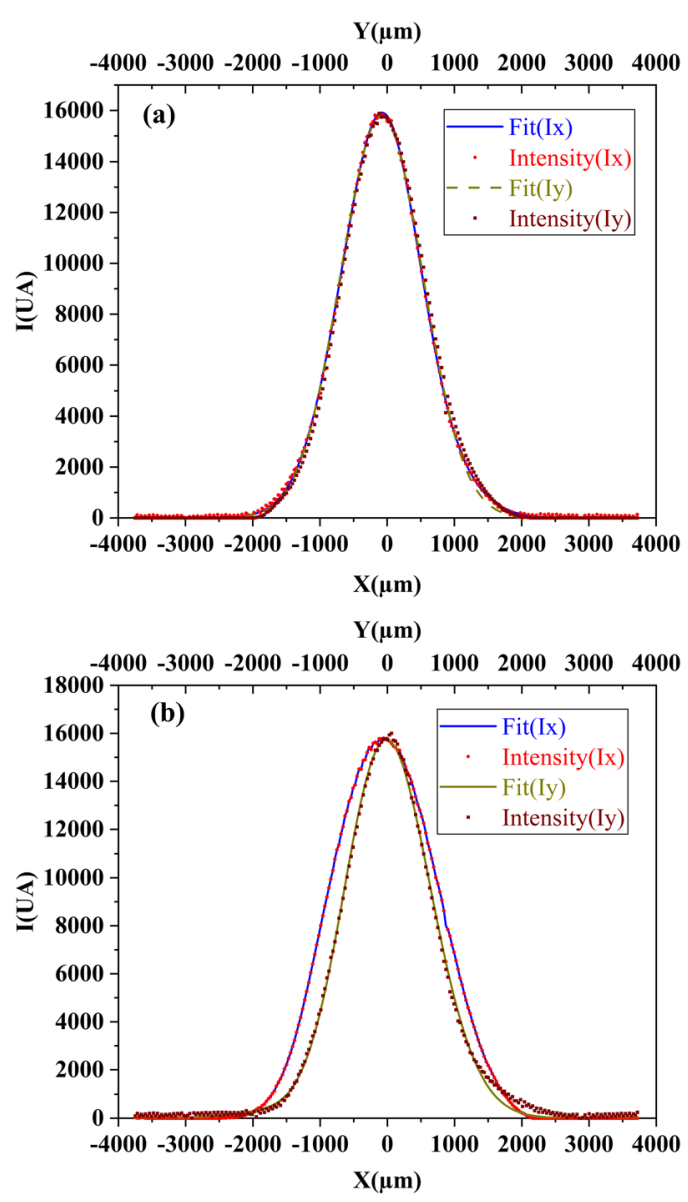

Figure 12. Measured Near field transverse intensity scan data and the Gaussian fit: (a) SMF, (b) major (x) and minor (y) axis of the hemi- ellipsoidal microlens (M1).

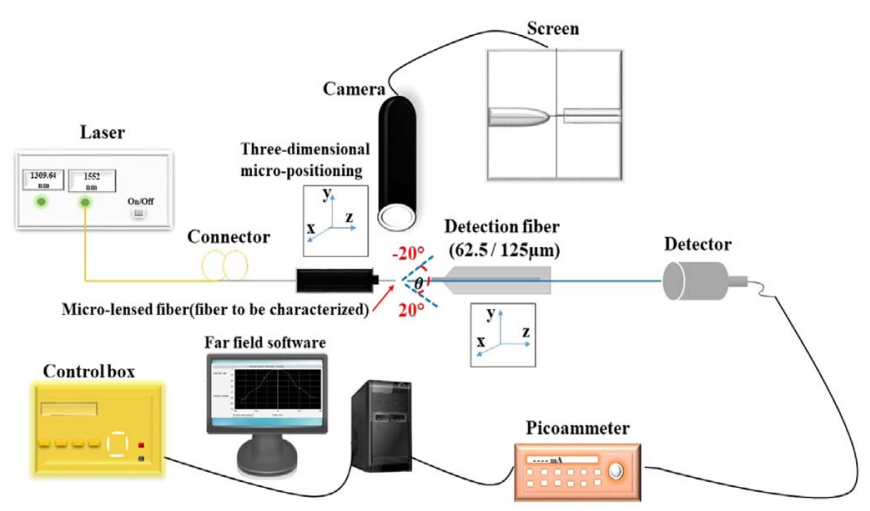

Figure 13. Illustration of the far-field measurement setup.

\subsection{Description of the far field measurement method}

Far field measurement technique is used to measure the MFD far away from the Rayleigh area. The fiber far-field profile setup consists of $1.552 \mu$ m laser sources, a 62.5/125 $\mu \mathrm{m}$ multimode fiber connected to an InGaAs detector. The measurement is performed by angularly moving this fiber in a range depending on the beam divergence $\left(-20^{\circ}\right.$ to $+20^{\circ}$, in this work) at a distance from the microlens output which is estimated, according to the expected MFD and the laser used, to be in far field. The microlens output is placed at the rotation center of the detecting fiber as shown in the schematic diagram in figure 13.

The far field light intensity (I) profile, is recorded versus this $\theta$ angle of emission for wavelength $1.552 \mu \mathrm{m}$ (figure 14). This measurement technique [24] allows to plot the far field distribution $I=f(\theta)$ and to calculate by several methods the MFD of the microlensed fibers from the measured far field curves [25]. 


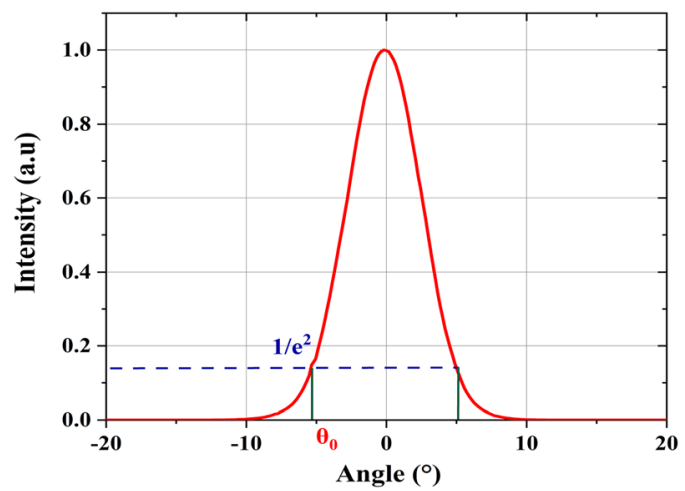

Figure 14. Far-field intensity distribution versus scanning angle for the M5 microlens along the major axis for wavelength $1.552 \mu \mathrm{m}$.

Table 4. Mode field diameter at the waist measured by far field method (1.552 $\mu \mathrm{m}$ lasers), for the M1 microlens with a measurement error OF $\pm 0.50 \mu \mathrm{m}$.

\begin{tabular}{|c|c|c|c|c|c|c|c|c|c|c|}
\hline & & & & & & & & Pet & & \\
\hline & & & & & & & & & & \\
\hline & & & & & & & & & & \\
\hline Micro-lenses & Lasers & $\mathrm{Ma}$ & $\mathrm{Mi}$ & $\mathrm{Ma}$ & $\mathrm{Mi}$ & $\mathrm{Ma}$ & $\mathrm{Mi}$ & $\mathrm{Ma}$ & Mi & MFD ratio \\
\hline & $(\mu \mathrm{m})$ & & & & & & & & & $\mathrm{Mi} / \mathrm{Ma}$ \\
\hline M1 & $(1.552 \mu \mathrm{m})$ & 79.49 & 60.39 & 10.05 & 8.76 & 9.71 & 8.76 & 10.08 & 8.33 & 0.86 \\
\hline M5 & $(1.552 \mu \mathrm{m})$ & 87.00 & 76.39 & 10.52 & 9.85 & 10.22 & 9.28 & 10.67 & 9.78 & 0.91 \\
\hline
\end{tabular}

Eff $\mathrm{A}=$ Effective area, Eff M D = Effective mode diameter, $\mathrm{MD}=$ Mode diameter, $\mathrm{MFD}=$ Mode field diameter, $\mathrm{Ma}=$ major axis $(\mathrm{x}$ direction), $\mathrm{Mi}=$ Minor axis ( $\mathrm{y}$ direction).

We thus obtain the MFD by measuring the angle $\theta_{0}$ corresponding to the $1 / e^{2}$ intensity maximum on the measured $I=f(\theta)$ curve (see the example in figure 12), or on a gaussian fit of the measured curve. The waist is calculated in the far field approximation by $w_{0}=\nu\left(\pi \cdot \operatorname{tg} \theta_{0}\right)$.

We also deduce the waist from the effective area by the inverse Hankel method or by the Peterman method [25, 27-29].

The resolution for a given mode field diameter to be measured depends on the core diameter of the multimode fiber connected to the detector and the distance between this multimode fiber and the microlens. In this example, this resolution is $\pm 0.5 \mu \mathrm{m}$ for the MFD measurement.

And for a given far field system the smaller the mode field diameter to be measured the wider the divergence angle of the beam, so the better the resolution.

Table 4 represents an example of the MFD at the waist location measured by the four methods (Effective area, Effective mode diameter, Mode diameter at Ima $/ e^{2}$ and Peterman method) for the case of the M1 and M5 microlenses with wavelength $1.552 \mu \mathrm{m}$.

Since there was not too much difference between the values measured by the four methods (on the left and right side of the Gaussian), these values were averaged to obtain the average MFD of the microlens (values of MFD shown in table 4).

Far-field measurement technique is possible but not the most suitable in this case of fairly large and above all elliptical mode diameters (since only one axis is measured at a time and the fiber must be positioned correctly on each axis).

But this method is also interesting because it allows to give additional informations on the profile ellipticity of these microlenses by transforming the far field data to the near field data and calculating the near field from far field.

To finish, the measurement technique in near fields and also in far fields are capable to show beam asymmetry in $\mathrm{x}$ and $\mathrm{y}$ directions offered by the hemi-ellipsoidal microlens as expected.

\section{Experimental measurements and theoretical calculations}

Table 5 compares the experimental major and minor axis MFD measured in near field (2nd and 3rd column) with theoretical ones (5th and 6th column) calculated thanks to the Kogelnik ABCD method [16, 17] for 


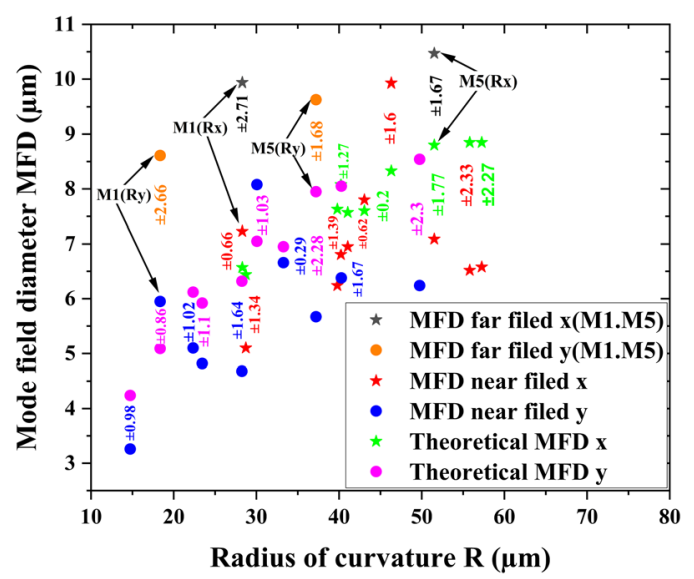

Figure 15. Theoretical and experimental mode diameters as a function of the radius of curvature of the microlenses for given thicknesses (values of table 2).

Table 5. Comparison between MFDs measured in near field and calculated theoretically in the two planes (X, Y) of the microlenses.

\begin{tabular}{|c|c|c|c|c|c|c|}
\hline \multirow[b]{2}{*}{ Micro-lenses } & \multicolumn{2}{|c|}{ Measured MFD Near field } & \multirow[b]{2}{*}{ MFDy/MFDx } & \multicolumn{2}{|c|}{ Theoretical MFD } & \multirow[b]{2}{*}{ MFDy/MFDx } \\
\hline & $\mathrm{Ma}$ & $\mathrm{Mi}$ & & $\mathrm{Ma}$ & Mi & \\
\hline M1 & 7.23 & 5.95 & 0.82 & 6.57 & 5.09 & 0.77 \\
\hline M2 & 6.52 & 6.38 & 0.97 & 8.85 & 8.05 & 0.90 \\
\hline M3 & 6,24 & 4.82 & 0.77 & 7.63 & 5.92 & 0.77 \\
\hline M4 & 9.93 & 8.08 & 0.81 & 8.33 & 7.05 & 0.84 \\
\hline M5 & 7.09 & 5.67 & 0.80 & 8.8 & 7.95 & 0.90 \\
\hline M6 & 7.80 & 4.68 & 0.60 & 7.60 & 6.32 & 0.83 \\
\hline M7 & 6.81 & 5.10 & 0.74 & 8.08 & 6.12 & 0.75 \\
\hline M8 & 5.10 & 3.26 & 0.63 & 6.44 & 4.24 & 0.65 \\
\hline M9 & 6.58 & 6.24 & 0.94 & 8.85 & 8.54 & 0.96 \\
\hline M10 & 6.95 & 6.66 & 0.95 & 7.57 & 6.95 & 0.91 \\
\hline
\end{tabular}

$\mathrm{MFD}=$ Mode field diameter $\mathrm{Ma}=$ major axis (x direction), $\mathrm{Mi}=$ Minor axis (y direction).

different ellipticity MFD ratios (column 4, 7), of the microlenses referenced in first column. For the calculation of these theoretical points of the MFD, we took the values of the thicknesses T of table 2.

Figure 15 illustrates the results obtained for the different hemi-ellipsoidal microlenses relative to values of mode field diameters MFD measured in near fields and calculated theoretically as a function of the radius of curvature of the microlenses for given thicknesses according to the two planes of the microlens.

We also compared the MFD $x$ and MFD of the M1 and M5 microlens measured in the far field with the values measured in the near field and calculated theoretically for the same microlens (examples shown in figure $15 \mathrm{MFD}_{x}$ far field and $\mathrm{MFD}_{y}$ far field).

We notice that the two ratios of the MFDs of the M5 microlens measured in far field (0.91), and calculated theoretically (0.90) are identical. The same for the M1 microlens measured in far field (0.86), and near field (0.82) with a small difference with the ellipticity ratio for the theoretical calculated MFD (0.77).

A difference is observed between the values of the MFDs measured in far fields by taking $\mathrm{MFD}_{\mathrm{x}}, \mathrm{MFD}_{\mathrm{y}}$, the mean values of the measurement by the four methods for the $\mathrm{M} 1$ microlens $\left(\mathrm{MFD}_{\mathrm{x}}=10,47 \mu \mathrm{m}\right),\left(\mathrm{MFD}_{\mathrm{y}}=\right.$ $9,63 \mu \mathrm{m})$ compared to those of near fields $\left(\mathrm{MFD}_{\mathrm{x}}=7.09 \mu \mathrm{m}\right),\left(\mathrm{MFD}_{\mathrm{y}}=5.67 \mu \mathrm{m}\right)$ and theoretical MFD $\left(\mathrm{MFD}_{\mathrm{x}}=8.8 \mu \mathrm{m}\right),\left(\mathrm{MFD}_{\mathrm{y}}=7.95 \mu \mathrm{m}\right)$.

This could partly be explained by the fact that the microlens transverse intensity mode profile is not perfectly Gaussian as pointed out with the secondary lobes in far field (figure 12), it's just an approximation, since it is not in near field, while only a Gaussian near field remains Gaussian in far field.

Figure 15 is quite complete and not so easy to understand since it gathers all the MFDs values obtained for a given radius of curvature in the two $\mathrm{x}$ and $\mathrm{y}$ directions of the microlenses. 


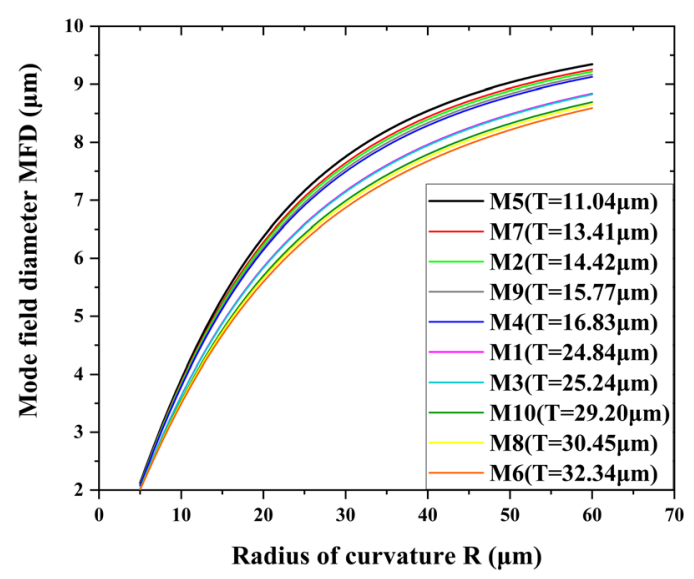

Figure 16. Theoretical mode field diameters as a function of the curvature radii for each thickness of the microlenses at $\lambda=1.55 \mu \mathrm{m}$.

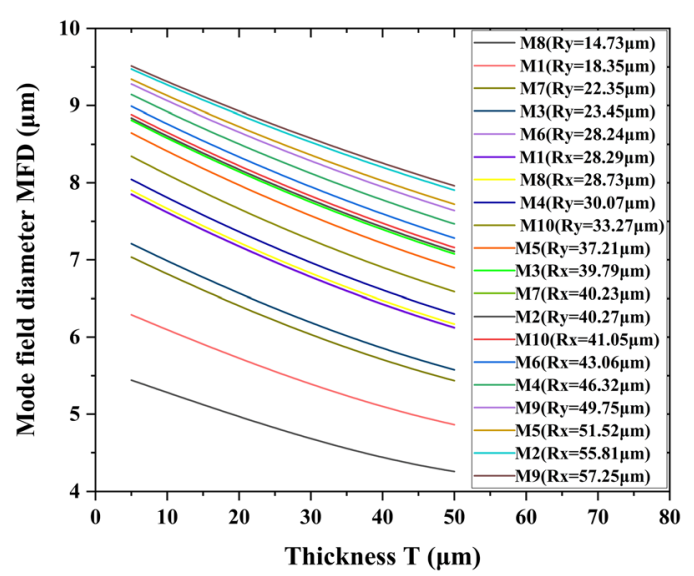

Figure 17. Theoretical mode field diameters as a function of the thicknesses for the radii of curvature Rx, Ry of the microlense (values of table 2).

But from table 5 and figure 15, it is clear that for the same values of the radii of curvature, different MFDs were obtained for different thickness values which offers a wide range of MFD plotted in figure 15.

According to the values of the mode field diameters shown in table 5 , it can be seen that the ellipticity ratios of the MFDs calculated theoretically resemble the values measured in near field with an error range between \pm 0 and \pm 0.1 (except for M6).

It is concluded, the radii of curvature $\mathrm{Rx}, \mathrm{Ry}$, the diameters Dx, Dy and also the thicknesses are decisive to dimension the microlenses with the desired ellipticity ratios. And this ellipticity ratio is very important since not only the mode field diameter do not usually match each other, but also the geometric shapes of the LD and the SMF are different (elliptical for the laser diode and circular for the SMF).

Moreover, the MFDs dimension remains to be optimized in addition to the ellipticity MFD ratios. The interest of fabricating hemi-ellipsoïdal microlenses instead of hemi-spherical ones depends on the coupling loss required for the application. A trade-off has to be found between the interest of improving coupling efficiency and advanced fabrication process. Figure 16 illustrates the variation of the theoretical waist of each microlens, calculated by the ABCD matrix method $[16,17]$ at a wavelength $\lambda=1.55 \mu \mathrm{m}$, as a function of the radii of curvature measured for given thickness values varying from 11.04 to $32.34 \mu \mathrm{m}$. As could be expected, it is shown that the MFD increases when the radii of curvature of the microlenses increases.

Figure 17 represents the theoretical values of the waist (2w = MFD) (calculated by ABCD matrix [16, 17]) as a function of the thicknesses of the microlenses in both minor and major planes for given values of the radii of curvature at $\lambda=1.552 \mu \mathrm{m}$.

According to these values of the radii of curvature Rx, Ry of each microlens manufactured by this technique, the values of the mode field diameters (MFD) are small when the thicknesses values are large as in the case of the 
M3 microlens: for a thickness of $25 \mu \mathrm{m}$, values of the mode field diameters varies in a range of 6.38 to $4.25 \mu \mathrm{m}$ (table 2) corresponding to the MFDs (near field) in the $\mathrm{x}$ and y planes respectively.

The theoretical values in figure 15 are plotted with the results shown in figures 16 and 17 . Even if it is obvious, these curves points out the interest of varying the thickness for a given radii of curvature and vice versa to achieve a wide range of MFD with this kind of microlenses. It helps to well understand the previous figure 15 which was quite rich.

\section{Conclusion}

This work consists of the realization of several micro-collimators with different hemi-ellipsoidal microlenses. The fabrication process is fully automated. It should be noted that the choice of the appropriate shape to solve the problem of mode mismatch between mode profiles of the laser diode and single mode fiber is necessary. A method to produce hemi-ellipsoidal microlenses on the end of an optical fiber is investigated. Microlenses with different dimensions and thicknesses are studied. This manufacturing technique also offers a wide variety of major and minor axis diameter (Dx and $D y$ ) ranging from $56.11 \mu \mathrm{m}$ to $120.7 \mu \mathrm{m}$ with different radii of curvature ( $R x$ and $R y$ ) ranging from $14.73 \mu \mathrm{m}$ to $57.25 \mu \mathrm{m}$ in the two planes of the microlens forming different ellipticity diameters ratios in a range of 0.68 at 0.84 and 0.51 to 0.86 for radii of curvature ones. The optical characterization of mode diameters of asymmetric beam laser diodes by injection of light and determination of the asymmetry of the beam at the microlens output are also discussed.

The proposed method allows to control the ellipticity ratio by adjusting the polishing speed during the mechanical micro-machining step to obtain optimal radii of curvature and thicknesses leading to mode field diameter in a range of $3.26 \mu \mathrm{m}$ to $9.93 \mu \mathrm{m}$ and elliptical MFD ratios in a range of 0.60 to 0.97 measured in near field. These ellipticity values are still rather low, but we believe that the method is promising for achieving more elliptical MFDs ratios. We trust that it is suitable for low cost, and reproducible fabrication process since it is quite simple.

In conclusion, the proposed new hemi-ellipsoid shaped fiber end structure is promising for achieving highcoupling efficiency between elliptical laser or waveguide beams and fibers optical communication applications and for many applications in other wavelength ranges as well. So, the aim remains to optimize the optogeometric parameters, not only the waist characterized in this work, but also the working distance where this waist is focused. That will subsequently be a second work. In this next work, the theoretical and measured working distances, coupling losses, of these new elliptical microlenses, in front of fibers and laser diodes as well as the positioning tolerances will be presented.

\section{Acknowledgments}

Financial supports from French and Algerian government: Ministry of Higher Education and Scientific Research and Campus France are greatly acknowledged for providing financial assistance to this Research Project in the framework of the Franco Algerian cooperation PROFAS B +2019 program.

\section{Data availability statement}

No new data were created or analysed in this study.

\section{ORCID iDs}

Nadjiba Boulaiche (iD https://orcid.org/0000-0001-9250-625X

Monique Thual (iD https://orcid.org/0000-0002-9944-938X

\section{References}

[1] Chao C K, Hu J Y, Hung S Y and Yang H H 2010 J. Mech 261

[2] Sakai J I and Kimura T 1980 IEEE Journal of Quantum Electronics QE- 1610

[3] Lu Y K, Tsai Y C, Liu Y D, Yeh S M, Lin C C and Cheng W H 2007 Opt. Express 151434

[4] He M, Yuan X, Ngo N Q, Cheong W C and Bu J 2003 Appl. Opt 4236

[5] Shah V, Curtis L, Vodhanel R S, Young W C and Bour D P 1990 J. Lightw. Technol 89

[6] Presby H M and Gile C R 1993 IEEE Photonics. Technol. Lett 52

[7] Edwards C, A Presby H M and Dragone C 1993 J. Light. Technol 112

[8] Modavis R and A, Webb T W 1995 IEEE Photonics. Technol. Lett 77

[9] Yoda H and Shiraishi K 2001 J. Light. Technol 1912 
[10] Yoda H, Endo T and Shiraishi K 2002 J. Light. Technol 208

[11] Irie Y, Miyokawa J, Mugino A and Shimizu T 1999 OFC/IOOC238-240 (in Tech. Dig.OFC/IOOC)

[12] Yeh S M, Lu Y K, Huang S Y, Lin H H, Hsieh C H and Cheng W H 2004 J. Light. Technol 225

[13] Yeh S M, Huang S Y and Cheng W H 2005 J. Light. Technol 231

[14] Huang SY, Yeh S M and Cheng W H 2005 in Tech. Dig. CLEO/CWN2 1473-75

[15] Liu Y D, Tsai Y C, Lu Y K, Wang L J, Hsieh M C, Yeh S M and Cheng W H 2011 J. Light. Technol. 296

[16] Mukhopadhyay S2016 JOpt46359-67

[17] Kogelnik H and Li T 1966 Appl.Opt 510

[18] Ziliang C, Weiping Q, Guocheng S and Wanjun W 2013 Sensors and Actuators A 20444

[19] Camou S, Fujita H and Fujii T 2003 Lab Chip 3 40-45

[20] Shao G, Qiu W and Wang W 2010 Microsystem Technologies 161471

[21] Yang C, Shi K, Edwards P and Liu Z 2010 Optics Express 1823529

[22] Yamamoto T, Fujii T, Nojima T, Hong J W and Endo I 2004 Proc, SPIE 4177

[23] Zaboub M, Guessoum A, Demagh N E and Guermat A 2016 Opt. Commun 366122

[24] Anderson W T and Philen D L 1983 J. Light. Technol LT 11

[25] Nguyen D.M, Le S.D, Brilland L, Coulombier Q, Troles J, Mechin D, Chartier T and Thual M 2010 ECOC 1 (IEEE)

[26] Nguyen D M, Blin S, Nguyen T N, Le S D, Provino L, Thual M and Chartier T 2012 App.Opt 514

[27] ArtigliaM C G, Vita D P, Potenza M and Sharma A 1989 J. Light. Technol 78

[28] Petermann K 1976 Electron.Lett 124

[29] Petermann K 1983 Electron. Lett. 19712 